\title{
Noire n'est pas mon métier ou un autre appel des Indigènes de la République
}

\section{Jacques Gérard Keubeung Fokou}

Le Noir, que Pap Ndiaye définit comme celui qui appartient à « un groupe de personnes ayant en partage l'expérience sociale d'être considérées comme noire» (23), reste sujet à problèmes au sein de la société française. Depuis l'esclavage et la colonisation, le Noir semble être marqué du sceau des stigmates et stéréotypes qui ont justifié ces entreprises. Je ferai l'économie des récits de voyages du quinzième siècle, des articles du Code Noir qui a aplani les sentiers pour l'infériorisation du Noir, et du discours des Philosophes des Lumières qui a soutenu la mise en esclavage de ces êtres établis comme une altérité parce que différents. L'esclavage a vu la déportation des Noirs du continent africain. Il sera suivi par la colonisation en tant qu'invasion par les puissances occidentales, dont l'objectif premier est de partager cette vaste terre riche en ressources matérielles. À chaque fois, les mêmes discours serviront de justificatifs. Près d'un demisiècle après ces évènements, en dépit des indépendances, des brassages de populations, et de la mondialisation qui réduit les frontières entre les êtres et les territoires, contribuant à l'émergence d'une identité cosmopolite, le Noir reste marginalisé et victime de fausses représentations. En France, pays des Droits de l'Homme et du Citoyen, ces représentations prennent à chaque fois des visages différents comme les synthétise Mame-Fatou Niang :

À partir des années 1980, les représentations des Noirs vont peu à peu s'orienter vers les questions d'immigration, d'intégration et d'assimilation. Les années 1990 voient l'émergence du « syndrome malien » avec la réification des figures de la mère immigrée analphabète, du père polygame, des garçons en décrochage scolaire et des filles victimes de l'autorité patriarcale. Dans l'espace et les discours publics, la figure du Noir est désormais associée à celle du mari polygame flanqué d'une horde d'enfants, à celle de l'adolescente franco-sénégalaise excisée à quelques encablures des Champs-Élysées, ou encore à celles des sans-papiers de l'église StBernard. Le Noir ce sont aussi « les bruits et les odeurs » qui hantent la France. (223-224)

Ces représentations sont des vecteurs de discriminations et d'exclusions. Face aux composantes du pouvoir d'État français qui utilisent ces stéréotypes pour les confiner aux marges de la société, les minorités françaises opposent très souvent manifestations et soulèvements. Les émeutes de l'automne 2005 constituent le point culminant de ces réactions des populations marginalisées qui reprochent à la société de ne pas leur céder un peu d'espace de visibilité. Dans le contexte qui a 
précédé ces émeutes, un groupe de citoyens français constitués en collectif dénommé les Indigènes de la République a rendu un appel dénonçant les discriminations et injustices dont ils sont les victimes au sein de la société française. L'appel des Indigènes de la République intervient en réaction à deux événements majeurs survenus dans la vie politique de la France en 2005, tout d'abord, la création du musée du colonialisme qui présentait la colonisation d'une façon favorable ; ensuite le vote par le parlement français de la loi du 23 février 2005 qui recommandait dans son article 4, alinéa 2, l'inclusion dans les programmes scolaires et son enseignement du rôle important de l'armée française dans les colonies. Pour d'aucuns, tel que le soutient Romain Bertrand, « elle est l'indice de la permanence, voire du regain d'activisme, de réseaux « Algérie française » ancrés dans des communautés « pieds-noirs » politiquement proches de la droite extrême et attachés à la réhabilitation publique de la geste de l'OAS (Organisation armée secrète). Pour d'autres, elle est le produit du laisser-aller d'une classe politique déliée de son électorat populaire, et donc insensible aux désarrois identitaires et au sentiment de mise à l'écart des enfants des « deuxième et troisième générations » de l'immigration en provenance des sociétés du Maghreb et de l'Afrique subsaharienne (Bertrand 29).

Ces deux événements suscitent l'indignation de certains Français, parmi lesquels Houria Bouteldja, Youssef Boussoumah et leurs compagnons qui rédigent l'appel des Indigènes de la République dans lequel ils soutiennent que la France a été et reste un état colonial qui doit entreprendre une décolonisation des esprits et des institutions. Le refus par la France d'entreprendre cette décolonisation des institutions et des esprits, selon les auteurs de ce manifeste, discrimine sa population issue des anciennes colonies d'Afrique. C'est à la lumière et dans l'esprit de ce manifeste que le présent article se propose de lire le livre Noire n'est pas mon métier, recueil des expériences de seize actrices françaises noires dénonçant les injustices et les discriminations qu'elles rencontrent dans l'univers du cinéma et du théâtre. Ces actrices sont Nadège BeaussonDiagne (Podium, Plus belle la vie, Bienvenue chez les Ch'tis), Mata Gabin (La valse des gros derrières ; Sexe, Gombo et beurre salé), Maïmouna Gueye (La première étoile, Mignonnes), Eye Haidara (C'est la vie), Rachel Khan (L'invitation), Aïssa Maïga (Bienvenue à Marly-Gomont; Bamako), Sara Martins (Un mensonge oublié ; Le poids des mensonges), Marie-Philomène NGA (Bienvenue à Marly-Gomont; Il a déjà tes yeux), Sabine Pakora (La fête des mères, Il a déjà tes yeux), Firmine Richard (Romuald et Juliette, Huit femmes), Sonia Rolland (Les pygmées de Carlos, Léa Parker), Magaajyia Silberfeld (Vagabonds), Shirley Souagnon (Le feu sacré), Assa Sylla 
(Bande de filles), Karidja Touré (Bande de filles; La Colle) et France Zobda (Meurtres en Martinique ; Toussaint Louverture).

Le postulat de base sur lequel j'entends construire cette étude est que Noire n'est pas mon métier se présente comme un autre appel des Indigènes de la République. Cela revient à prendre en considération le double paramètre du genre et de la race des auteures, ce qui évite de confondre le combat de ces femmes avec celui d'autres femmes qui dénoncent le sexisme du cinéma français. Elles sont femmes, noires et issues de l'immigration coloniale. Ensuite, et pour citer le texte de l'appel des Indigènes de la République, « discriminées à l'embauche, au logement, à la santé, à l'école et aux loisirs, les personnes issues des colonies, anciennes ou actuelles, et de l'immigration post-coloniale sont les premières victimes de l'exclusion sociale et de la précarisation » (site internet de l'association). La prise en compte de ces paramètres revient à poser également que « la figure de l'indigène continue à hanter l'action politique, administrative et judiciaire ; elle innerve et s'imbrique à d'autres logiques d'oppression, de discrimination et d'exploitation sociales » (Appel des Indigènes de la République, site de l'Association). En conséquence, les Indigènes de la République concluent : «La république de l'Égalité est un mythe. L'État et la société doivent opérer un retour critique radical sur leur passé-présent colonial. Il est temps que la France interroge ses Lumières, que l'universalisme égalitaire, affirmé pendant la Révolution Française, refoule ce nationalisme arc-bouté au 'chauvinisme de l'universel', censé 'civiliser' sauvages et sauvageons » (site de l'Association). Noire n'est pas mon métier, tentative de prise de parole des actrices noires, à qui l'industrie cinématographique refuse la visibilité à travers des rôles décents, est aussi comparable au combat de la femme noire triplement subalterne et condamnée à une bataille permanente qui vise à s'approprier une humanité qui lui est refusée afin d'accéder à ce que Jacques Rancière appelle le partage du sensible et qui se définit comme suit : « le partage du sensible » est « la façon dont les formes d'inclusion et d'exclusion qui définissent la participation à une vie commune sont d'abord configurées au sein même de l'expérience sensible de la vie » (Palmieri 34). De façon plus concrète,

C'est le procédé qui définit la gestion de la vie au sein d'une communauté en tenant compte de tous. C'est ce système d'évidences sensibles qui donne à voir en même temps l'existence d'un commun et les découpages qui y définissent les places et les parts respectives. [...] Cette répartition des parts et des places se fonde sur un partage des espaces, des temps et des formes d'activité qui détermine la manière même dont un commun se prête à participation et dont les uns et les autres ont part à ce partage [...]. [C'est] un découpage des temps et des espaces, du visible et de 
l'invisible, de la parole et du bruit qui définit à la fois le lien et l'enjeu de la politique comme forme d'expérience. (Site internet Multitudes)

Noire n'est pas mon métier pose le double problème de l'absence et de la présence des Noirs dans l'espace public français. Dans l'introduction au livre, Aïssa Maïga, actrice et initiatrice du projet, pose la question suivante : « Pourquoi autant de femmes et de jeunes filles talentueuses, issues d'Afrique et d'Outre-mer, qui maitrisent leur art, cinéma, théâtre, parfois chantent, écrivent, semblent rester irrémédiablement invisibles, ignorées ? Exclues de l'immense majorité des opportunités artistiques d'un pays pourtant doté d'une véritable industrie culturelle » (16). Afin que cette question ne soit pas éludée ou prise comme une vaine revendication sans fondement, l'actrice dresse un état des productions artistiques françaises :

Plus de trois cents films français sont produits chaque année. L'on ne compte plus les festivals de cinéma et de théâtre en France. Les Molières récompensent chaque année des dizaines de pièces. À la télévision, il n'y a jamais eu une telle production de séries. Et pourtant, il subsiste un vide retentissant en termes de représentations de la réalité sociale, démographique, ethnique française. Comment les réalisateurs et réalisatrices observent-ils ce vide ? En ont-ils conscience ? Se sentent-ils en phase avec la société dans laquelle ils vivent? Sont-ils toujours dans cette dichotomie que j'ai pu observer au début des années 2000 ? Le cœur à gauche, prompts à militer pour les droits des sans-papiers hier et des réfugiés aujourd'hui ; mais difficilement prêts à offrir une narration incluant les « autres », les nonBlancs, dans leurs films, téléfilms et pièces de théâtre ? (8)

Tandis que l'absence des non-Blancs dans les productions cinématographiques et théâtrales pose le problème d'éthique républicaine et interpelle les responsables sur la sous-représentation des actrices noires en nombre dans ces productions, la question de la présence interroge les rôles qui leur sont attribués et qui perpétuent les représentations abjectes du corps noir. Ainsi, le livre soulève avant tout la question du regard de l'autre sur le corps noir féminin. Le corps de la femme noire apparait à la fois fantasmé, caricaturé, méprisé et désiré. Ces multiples déclinaisons du désir/mépris du corps de la femme noire sont véhiculées à travers l'image et le stéréotype. Le désir qui nait chez l'interlocuteur de Beausson-Diagne à la vue de son postérieur (13), les représentations négatives de la femme africaine qu'incarne Marie-Philomène Nga (65) exemplifient cette situation.

Dans « De l'imagerie culturelle à l'imaginaire », Daniel Henri Pageaux soutient :

Toute image procède d'une prise de conscience, si minime soit-elle, d'un Je par rapport à l'Autre, d'un Ici par rapport à un Ailleurs. [...] l'image est la représentation d'une réalité culturelle au travers de laquelle l'individu ou le groupe 
qui l'ont élaborée (ou qui la partagent ou qui la propagent) révèlent et traduisent l'espace culturel et idéologique dans lequel ils se situent. (135)

Les images de la femme noire et les stéréotypes à l'aide desquels on la désigne, sont réducteurs et la pose comme un être inférieur. Ce regard trouve son origine dans le passé colonial de la société française. Noire n'est pas mon métier s'inscrit dans la lignée des auteurs du collectif Décoloniser les Arts. Dans une publication du 7 novembre 2016 intitulée « la nécessaire décolonisation des arts et des imaginaires », les membres du collectif justifient leur existence au fait que les domaines de l'art « sont traversés de tensions et de logiques coloniales, imprégnés et embarrassés par l'histoire et l'idéologie coloniale. [Pour ce collectif], il est urgent de travailler à déconstruire et à remettre en cause ces systèmes de hiérarchie et d'inégalités, tout comme à décoloniser en profondeur nos imaginaires » (site internet du collectif). Analyser les images et les mécanismes d'invisibilisation de la femme noire dans le secteur du cinéma participe de cette logique de décolonisation des imaginaires. Cela implique un effort de compréhension de l'imaginaire français, c'est-à-dire les façons dont cette société se voit, se définit et se rêve (Pageaux 136). De manière pratique, étudier l'image de la femme noire, c'est vouloir comprendre, comme le suggère Pageaux, « ce qui la construit, ce qui l'authentifie, ce qui la rend, le cas échéant, semblable à d'autres ou originale. [...] Étudier comment s'inscrivent diverses images de l'étranger [puisqu'elle est ici considérée comme telle], c'est étudier les fondements et les mécanismes idéologiques sur lesquels se construisent l'axiomatique de l'altérité, le discours sur l'autre » (137). Dire que ces femmes sont considérées comme des étrangères ne relève pas de l'affabulation. En effet, Gabin Mata, actrice et une des auteures, trouve absurde l'idée que « dans les quatre secondes qui suivent la première rencontre on te fait bien comprendre que pèse sur toi une présomption de non-appartenance à la France » (23), ce qui va à l'encontre des lois françaises qui ne reconnaissent pas la question des races, mais qui pose cependant la délicate question de la francité.

L'absence des femmes noires dans les productions cinématographiques ou leur présence à travers les rôles dégradants renforce leur sentiment d'exclusion de la nation française. La place qui semble leur être réservée, est dans l'arrière-cour de la république. L'exclusion des actrices, que j'assimile dans cette étude aux Indigènes de la République, se décline ici par l'attribution des stéréotypes qui sont des termes dégradants, réducteurs et déshumanisants. Le stéréotype marquait en colonie la différence de statut entre les Citoyens, détenteurs de tous les droits, et les Indigènes ou sujets de l'empire. Dans De l'indigène à l'immigré, Pascal Blanchard et Nicolas Bancel 
soulignent que « les caractéristiques physiques [du Noir], caricaturées - les yeux en boule de loto, les lèvres lippues, les dents toutes dehors, le nez exagérément épaté -, sont associées à l'idée d'infériorité, souligné par le langage 'petit-nègre', signe 'évident' de l'incapacité des Noirs à assimiler la culture française »(21). La résurgence de cet appareil discursif discriminant à l'intérieur de la république témoigne de ce que l'indigène a survécu à la république et que le passé est éternellement présent pour ces Français. Comme à l'époque coloniale et à travers l'iconographie ${ }^{1}$ de ladite période, le colon s'illustrait par l'usage d'un discours et d'une posture paternaliste qui infantilisait le Noir. Noire n'est pas mon métier dénonce l'emploi des propos paternalistes que ce soit par des réalisateurs ou des collègues sur les plateaux de tournage et en dehors. Dans le livre, les exemples comme «ça va ma petite Nadège » (13), salutation lancée à l'endroit de l'actrice et autre co-auteure Nadège Beausson, illustre le mépris affiché par les acteurs français à l'endroit de leurs collègues Noirs et réintroduit le fait colonial dans les relations entre des citoyens jouissant en principe des mêmes droits et prérogatives au sein de la République. À ces propos paternalistes, l'actrice évoque l'alliance qui est faite entre elle et l'Afrique au cours des castings comme celui où le directeur lui pose la question suivante : « vous parlez africain ?» (13). Tout d'abord, il n'existe pas une langue africaine. Ensuite, l'Afrique est un continent qui regroupe une cinquantaine de pays. Nier ce fait ne peut être considéré comme de l'ignorance ou de la mauvaise foi. Les raisons de cette négation soulignent les réminiscences d'une mentalité colonialiste qui laisse penser que les Noirs viendraient d'un village dénommé Afrique où toutes les populations auraient en commun la même langue.

Refuser à ces actrices leur francité en les établissant comme Africaines vise surtout à leur reconnaitre des défauts attribués aux populations africaines et qui ont justifié la colonisation de ce continent, c'est-à-dire leur caractère primitif, bestial et barbare. Sabine Pakora, co-auteure, écrit à ce propos : «On me renvoyait vers des références aux peuples primitifs, à l'Afrique (continent dans lequel je n'avais pas vécu), on imaginait que j'avais forcément le rythme dans la peau, on me prêtait une agilité et des aptitudes physiques et corporelles particulière » (77). Mata évoque la référence qui est faite aux fauves de l'Afrique. La description de l'expérience d'un soir passé aux côtés d'un acteur français célèbre confirme que la femme noire est toujours perçue dans l'imaginaire de certains hommes comme un fauve sexuel. Le discours empreint de sensualité que retranscrit Gabin dans le livre le témoigne : « Oh ma gazelle blabla-bla, ma panthère, j’entends les tam-tams de l'Afrique, la chaleur de la savane, ma tigresse blabla-bla, je serai ton lion, ton taureau 
et toi nue, oh l'odeur de la jungle africaine, toi la calipige, oh tu me fais rugir, oh ma gazelle africaine blabla-bla » (13). L'exclusion de ces actrices par l'érotisation et la bestialisation des corps rappellent la figure de la Vénus Hottentote, Saartje Bartman. Parlant de cette jeune Sud-Africaine, Gilles Boëtsch et Pascal Blanchard écrivent :

The history of the Hottentot Venus is also the history of a change in scientific knowledge, from the cabinet of curiosity to the museum. It marks a shift in how the other was displayed, from private showings to public exhibits. The Hottentot Venus, a woman who was presumed to be afflicted by deformity, who was considered to come from a strange race, can be seen as a point of articulation between freak shows and ethnic shows. (192-193)

Considérée par les théories racistes comme faisant partie d'une race intermédiaire entre l'humain et l'animal, la Vénus Hottentote résume le regard posé sur la femme noire. Les actrices et auteures de Noire n'est pas mon métier sont à la fois objets de plaisir et sujet de dégoût, tout comme ce fut le cas pour la Vénus Hottentote.

Dans l'imaginaire français, la femme noire est également perçue comme un être opprimé. Elle est à la fois la femme au foyer, seule ou flanquée d'une coépouse. C'est elle qui subvient aux besoins du couple, au contraire de l'homme qui s'offre toutes les libertés. La femme noire, tout comme celle arabe, est un personnage de l'intérieur. Elle n'a pas accès à l'espace public. Aux représentations du jeune garçon délinquant, voyou, dealer de drogue, s'opposent celles de la femme séquestrée, ménagère pour celle qui travaille, de la jeune fille excisée et les multiples variations de l'immigré (Niang 223). C'est aussi dans ces représentations que les réalisateurs et scénaristes puisent la matière pour faire des rôles qu'ils attribuent aux Noir(e)s dans leurs différents films. De nombreux films ramènent la femme à des nationalités précises et avec des mêmes caractéristiques. Bande de filles (2014) de Céline Sciamma, Banlieusards (2019) de Kerry James et Les Misérables (2019) de Ladj Ly n'échappent pas à cette logique. C'est l'expérience que fait Marie-Philomène Ngah à qui on apprend que « la femme noire ne porte que des boubous et des sandales [...] qu'elle ne peut être que Malienne ou Sénégalaise, ne parle pas correctement français ou alors avec un fort accent à la Michel Leeb, habite un HLM insalubre avec un fils délinquant» (72). L'image de la femme analphabète ou qui s'exprime dans un français approximatif est une réplique du stéréotype colonial du « nègre » « y a bon banania ». Poser que le français ne peut être bien parlé par une catégorie de sa population revient à soutenir que ces personnes sont différentes. Il s'établit dès lors une scission entre « eux » et " nous ». Poser la différence entre Nous et les Autres ne fait pas forcement problème. Le problème qui se pose ici, 
est le contenu qui est mis dans l'élément de différence ou ce qu'implique cette différence, c'est-àdire l'incapacité d'avoir part au récit national ou d'accéder à l'espace public comme c'est le cas pour les actrices de Noire n'est pas mon métier. Selon Pageaux, « le stéréotype pose de manière implicite une constante hiérarchie, une véritable dichotomie du monde et des cultures » (140). C'est dire qu'il disqualifie et discrimine.

Noire n'est pas mon métier renseigne sur le fait que persiste dans l'imaginaire français l'idée selon laquelle « être noir » signifie infériorité, paresse et nullité. L'un des corollaires de cette préconception est leur exploitation par les acteurs économiques. Être noir pour un employeur signifie donc une personne réduite à certaines tâches. C'est ce dont héritent les actrices et auteures de Noire n'est pas mon métier. La présence de ces femmes sur les écrans n'est nécessaire que pour représenter les rôles de NOIRES, c'est-à-dire le rebut de la société. Pakora affirme qu'elle et ses consœurs représentent à l'écran et au théâtre uniquement des prostituées, des femmes sans papiers, des marâtres cupides malintentionnées, des femmes africaines à l'humeur joviale, folklorique, ridiculisées :

Je joue toutes les déclinaisons de la Mama et de la putain africaines; des personnages hauts en couleurs sans capital intellectuel ou économique. Mama, Fatou, Alimata, Fanta... Mes personnages construisent une image de l'autre purement exotique. Ils apparaissent comme des parenthèses anecdotiques : ils représentent des instants de respiration, de relâchement soudain, où s'exprime une truculence exacerbée, comme un clown personnifié qui apparait et canalise les tensions, les angoisses, les peurs et les pulsions. (75)

Porteur d'une définition de l'Autre qui se veut définitive et atemporelle, consacrant de fait la hiérarchie entre Nous et les Autres, le stéréotype devient le pilier fondamental du processus de discrimination et d'invisibilisation du Noir dans l'univers du cinéma, du théâtre et, de manière générale, de l'espace public.

À travers l'attribution de rôles dégradants et la représentation d'une certaine image des Noires, les films français renouvellent à leur manière les «zoos humains », c'est-à-dire l'exposition des indigènes dans des cages tels des animaux comme ce fut le cas lors de l'exposition coloniale internationale de Paris en 1931. Ces expositions ont contribué à ancrer chez l'Occidental la tendance à l'animalisation du Noir. Comme dans les zoos humains, les personnages que les actrices noires incarnent à l'écran, déprécient l'humanité de l'homme noir. La représentation qui est faite du corps noir à travers divers rôles décriés par les actrices, donnent à voir au reste de la communauté noire l'image qui est la leur au sein de l'imaginaire français, et n'offrent pas de 
modèles aux générations futures. Le rôle de Noire, condensé de stéréotypes déshumanisants et racistes, fixe les limites à l'intérieur duquel évolue chaque Noire qui s'aventure dans le monde du spectacle. Plus important encore, la discrimination par l'attribution des rôles dégradants pour ces femmes noires établit les limites que les autres ont fixées pour elles. L'expérience de Karidja Touré, l'une des quatre héroïnes noires de Bande de filles de Celine Sciamma, est édifiante. Très tôt, elle comprend que le milieu du cinéma ne lui est pas accessible en tant que jeune fille noire. En témoigne son interprétation des affiches de castings : «Quand il était écrit "jeune fille de 13 à 18 ans', je savais que ce n'était pas pour moi : je l'avais vite compris parce que sinon l'annonce était dirigée différemment : 'cherche jeune fille de 13 à 18 ans, noire'. S'il n'était rien précisé, alors le rôle était forcément pour une Blanche » (106).

L'auto-disqualification qui caractérise Touré est un sentiment que partagent toutes ces actrices et, par conséquent, renforce leur désappartenance à la France. La remise en question permanente de la francité de ces « français d'origine contrôlée », pour parler comme Jean-Thomas Ceccaldi et Mustapha Kessous, est l'un des facteurs importants qui justifient le ghetto économique dans lequel se retrouvent les communautés noires et arabes. Sur le plan économique, la discrimination dont sont victimes les actrices pose la question de l'accès aux fruits de la croissance. Le refus pour une actrice d'interpréter un rôle qu'elle juge dégradant, peut signifier le chômage ou même l'abandon de la pratique du métier qui est le sien. Firmine Richard, doyenne des actrices françaises noires, témoigne de la grande différence salariale qui existe entre les Noires et leurs collègues blanches : «Dans une comédie à succès dans laquelle j'ai tourné récemment, nous étions quatre comédiennes principales. J'ai appris que l'une d'entre elles - et je ne parle pas de l'actrice vedette - était payée cinq fois mieux que moi, pour un nombre de jours de tournage équivalent. Elle-même était choquée de découvrir la différence de salaire entre nous deux » (83).

Incarner un personnage met certainement à l'abri du chômage. Cependant, ce personnage transforme ces actrices, figures publiques auxquelles s'identifient des milliers de jeunes. Ces personnages ridicules, plutôt que d'être des modèles à suivre et des sources d'inspiration pour les jeunes de la communauté noire sont des vecteurs de honte. L'exposition de ces tares supposées du Noir contribue à légitimer la mise à l'écart des Noirs des espaces de visibilité. Cela s'interprète également comme une mise volontaire du Noir à la marge en ce sens qu'il est non seulement inapte à accéder à la civilisation, mais, en plus, qu'il semble se complaire dans la situation de marginal. Avoir un rôle dans un film ou dans une pièce de théâtre devient un handicap pour celle qui l'incarne 
en ce sens que l'acteur ou l'actrice devient vecteur d'une certaine image qui, au final, jette le discrédit sur sa communauté. La présence d'une actrice noire dans un film est presque toujours un « acte politique » et un « acte militant» (Haidara 34) de la part de chaque metteur en scène ou de chaque réalisateur. La présence comme l'absence devient sujette à problème et l'acteur/l'actrice parle plus qu'il ne dit mot. Les actrices de Noire n'est pas mon métier souffre de cette situation. Pour Eye Haidara, leur « présence ne doit pas être vue comme un acte de revendication. Un acteur n'a qu'une seule vocation, celle de jouer. [...] Je veux juste qu'on arrête de nous regarder et de faire comme si on avait déjà parlé alors qu'on n'a pas ouvert la bouche. Je voudrais être une actrice banale » (37). Au final, l'actrice qui est censée briller sous le feu des projecteurs, illuminer la vie des cinéphiles et de ses admirateurs, préfère paradoxalement rester inaperçue. Didier Fassin souligne :

La discrimination provoque un sentiment pénible et sa reconnaissance en tant que telle fait du sujet la victime d'une injustice. C'est à ce titre qu'il peut réclamer ses droits, mais c'est aussi sous cette condition qu'il peut légitimer sa souffrance. L'ambiguïté de cette double subjectivation-comme sujet de droit et comme sujet de la souffrance - et sa tendance à produire une forme de victimisation - se manifestent dans le contraste entre l'espace bien plus grand que le second occupe par rapport au premier, à la fois parce que l'administration de la preuve, aussi libérale serait-elle, n'en reste pas moins soumise à des règles qui restreignent l'expérience concrète de la discrimination et parce que les victimes de ses effets ne sont pas nécessairement prêts à en demander la condamnation ou la réparation devant les tribunaux. (418-419)

En se constituant comme des dénonciatrices des discriminations dont elles sont victimes, les actrices-auteures de Noire n'est pas mon métier souffrent d'une double peine. L'acte d'écrire n'est pas thérapeutique. Au contraire, il surexpose les victimes qui se retrouvent devant le tribunal des lecteurs en tant que victimes et plaignantes en même temps. Toutefois, l'acte d'exposition des discriminations peut se lire comme un début de solution pour que les actrices noires bénéficient des mêmes chances que leurs collègues blanches.

Discriminer les actrices noires revient également à les priver d'une certaine parole. Le message que véhicule le personnage prostitué ou la mère qui s'exprime dans une langue française approximative n'a pas d'écho, si ce n'est celui de susciter du rire ou de la pitié. En se limitant à ne représenter les Noires qu'à travers ces rôles, le cinéma français refuse de contredire les stéréotypes en montrant des Noir (e) s qui exercent des métiers honorables. Le monde du cinéma refuse ainsi 
de participer à l'émergence d'une société française égalitaire et inclusive que les actrices de ce livre appellent de leurs vœux.

Le livre laisse toutefois transparaitre un espoir qu'expriment les jeunes actrices comme Karidja Touré et Assa Sylla, deux héroïnes du film Bande de filles. A travers ce film, les jeunes actrices ont vécu leur « rêve de cinéma » (106) et prouvé aux nombreuses adolescentes noires que ce n'était pas tout à fait impossible pour des jeunes filles noires de devenir actrice en France : « Depuis la sortie de Bande de filles, je vois des changements. Déjà, il y avait cette affiche de nous, dans les rues de Paris. Quatre filles noires, jeunes, face à l'objectif s'étalaient sur les murs des villes. Une fille d'aujourd'hui aura plus d'exemples sous les yeux que moi, quand j'avais 17 ans, elle aura vu d'autres actrices black françaises, elle aura la preuve que c'est possible » (Sylla 104). Pourtant, Mame-Fatou Niang dément le projet de Sciamma qui prétend aller à l'encontre des stéréotypes en mettant en scène exclusivement des jeunes filles noires : «Bande de filles met en scène des corps qui sont à l'intersection de marginalités de genres, de race, de classe et d'appartenance géographique. Replacer ces identités périphériques dans un noyau universaliste requiert une réflexion qui prenne aussi bien en compte les représentations contemporains que leurs lignées historiques »(217-218). Sciamma retombe curieusement dans la représentation des stéréotypes, car son film remet en scène les personnages de la mère célibataire et du garçon dealer qui martyrise sa sœur ou encore des jeunes filles désœuvrées en décrochage scolaire. La solution reste en définitive entre les mains des victimes.

En poussant le cri de révolte comme le firent les Indigènes de la République à travers leur appel, les actrices et auteures de Noire n'est pas mon métier dénoncent la grave injustice qu'elles subissent au quotidien. L'interpellation des décideurs de l'industrie cinématographique, du théâtre et de la société française qui ne serait pas prête à accepter les Noirs dans un rôle est primordiale. On ne peut ignorer le travail du Collectif Décoloniser les arts qui œuvre pour une égalité des chances pour les artistes issus des minorités. Cependant, les actrices victimes de discrimination se doivent de travailler à s'imposer pour offrir à leurs semblables des modèles et démentir les stéréotypes qui servent à leur refuser l'accès aux productions culturelles françaises. Pakora interpelle ses consœurs et les acteurs français noirs à copier l'exemple de la communauté afroaméricaine : « nous avons besoin de sociétés de production, de scénaristes et de réalisateurs ou réalisatrices issus de la communauté afro-diasporique pour proposer d'autres types de héros ou d'héroïnes, qui transcendent les clivages sociaux et raciaux habituels, et développer un regard 
critique sur les rapports de domination » (79). L'émergence de figures importantes comme France Zobda, Ladj Ly ou encore Kerry James lui donne raison. Ces dernières se retrouvent en amont des projets et non plus en aval. Il leur appartient désormais de donner le change, de donner aux jeunes minorités des représentations positives auxquelles ils/elles peuvent s'identifier. C'est l'objectif que se fixe Zobda en tant que productrice : " raconter enfin nos imaginaires, nos histoires, notre histoire, proposer un regard DE la diversité et non SUR la diversité » (116).

\section{Bibliographie}

Bancel, Nicolas et Pascal Blanchard. De l'indigène à l'immigré. Paris : La Découverte, 1998.

Bertrand, Romain. «La mise en cause(s) du «fait colonial». Retour sur une controverse publique ». Politique africaine 102.2 (2006). 28-49.

Boëtsch, Gilles et Pascal Blanchard. «From Cabinet of Curiosity to the 'Hottentot Venus'. A Long History of Human Zoos ». The Invention of Race. Scientific and Popular Representations. Dir. Nicolas Bancel, T. David et D. Thomas. New York : Routledge, 2014. 185-194.

Fassin, Didier, « L'invention française de la discrimination ». Presses de Sciences Po 52 (2002). 403-424.

Kessous, Mustapha et Jean-Thomas Ceccaldi. Français d'origine Contrôlée. Infrarouge/France Télévision, 2014.

Maïga, Aïssa, dir. Noire n'est pas mon métier. Paris : Seuil, 2018.

Mouzet, Aurélia. «Les acteurs noirs du cinéma français : de l'ombre à la Lumière ?». Voix Plurielles 14.2 (2017). 50-61.

Ndiaye, Pap, La condition noire. Essai sur une minorité française. Paris : Calmann- Lévy, 2008.

Niang, Mame-Fatou. Identités françaises. Leiden : Brill, 2019.

Pageaux, Daniel-Henri. « De l'imagerie culturelle à l'imaginaire ». Précis de littérature comparée. Dir. Pierre Brunel et Yves Chevrel. Paris : PUF, 1989. 133-161.

Palmiéri, Christine. « Jacques Rancière : 'Le partage du sensible' ». Revue d'art contemporain 59 (2002). 34-40.

Parti des Indigènes de la République. http://indigenes-republique.fr/le-p-i-r/appel-des-indigenesde-la-republique/https://www.multitudes.net/Le-partage-du-sensible/ 
Rancière, Jacques. « 'Nouveau millénaire, défis libertaires', 'Le Partage du sensible', Entretien avec Jacques Rancière». Alice 2 (1999). http://www.multitudes.net/Le-partage-dusensible/

\begin{abstract}
Note
${ }^{1}$ On peut évoquer ici les publicités comme celle de la marque de riz Uncle Bens qui, pour vanter sa qualité, met en évidence la gourmandise du "nègre »; celle du café au chocolat $Y$ a bon banania qui s’inscrit dans le même registre ; enfin celle du détergent Woolite qui, pour montrer son efficacité suggère le blanchiment de la peau noire qui serait sale et laide. Il existe de nombreuses images caricaturant les lèvres, les yeux et les fesses des Noir(e)s à l'excès.
\end{abstract}

